\section{Digital Imaging in K-12 Biology}

James Ekstrom, Phillips Exeter Academy

http://science.exeter.edu/jekstrom/default.html

$\mathrm{K}-12$ instruction in biology has traditionally taken a very descriptive approach. This is in marked contrast to quantitative as well as qualitative way of looking at things in physics and chemistry. This qualitative/descriptive approach even extends into the laboratory portion of the biological course. One way to introduce a more quantitative approach is in the microscopy portion of the biology curriculum. Because cellular structure is primarily a microscopic province it makes sense to introduce students to the different microscopic tools such as TEM and SEM, as well as the light microscope that are used to investigate cell structure. It is easy to quantify microscopic work and the light microscope is the principle, if sometimes only instrument, found in biology classrooms.

A typical introduction to the microscope can involve a measurement of the "field of view," as well as getting use to the various controls found on the instrument. If the lowest power student objective is $4 X$ and the ocular $10 X$ this measurement can occur with a fair degree of accuracy using a $6^{\text {" }}$ mass-produced plastic ruler that also has a metric edge to it. Using a higher power objective would involve mathematically calculating what the field would be or using an inexpensive $\$ 15.00$ ) micrometer. Once the student makes these calculations for $40 \times(4 \times \times 10 X)$,
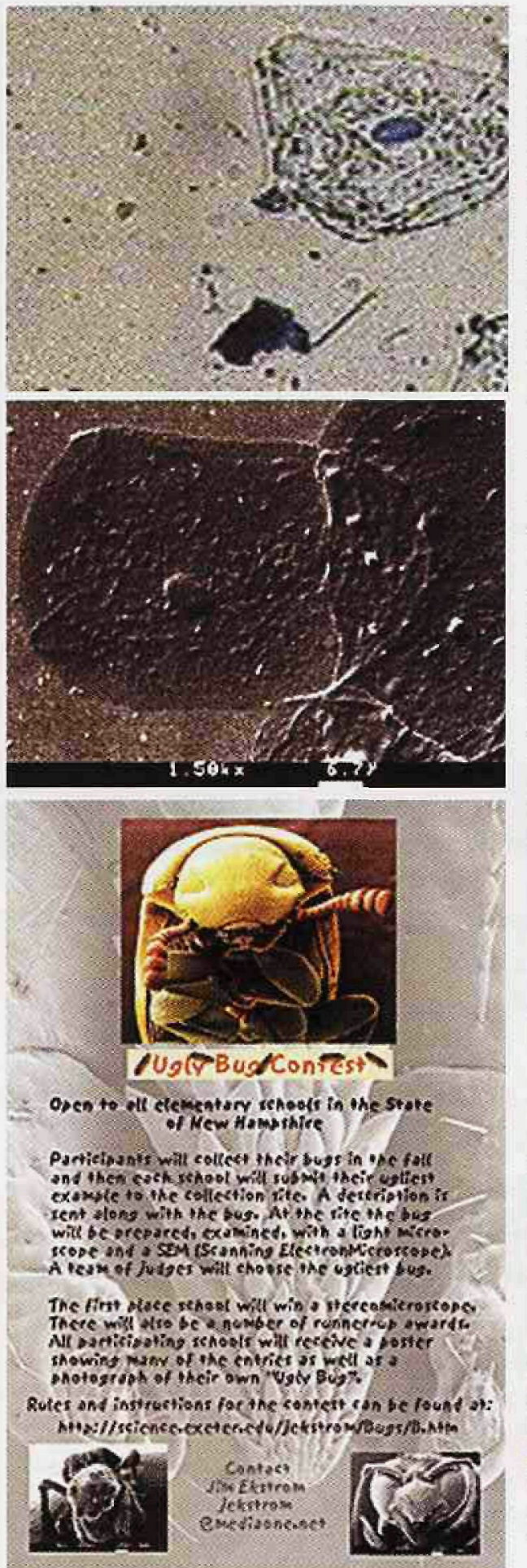

Figure 1 Top, Figure 2 Center, Figure 3 bottom
$100 \mathrm{X}$ and $400 \mathrm{X}$ they can record them and keep them witn tne microscope. At some future point if a "wee beastie" should occupy one-half of their field under $100 \times$ then they would have an approximate size of the object.

The advent of inexpensive digital photography allows the instructor to carry out a more sophisticated approach to this exercise. Digital images of the three fields of magnification can be stored as calibrations for that microscope. A subsequent microscopic image can be digitized and the reference scale for that magnification can be cut and pasted on the image.

[Figure 1] Cheek cells stained with methylene blue that has been photographed with the relevant scale pasted on the image. This calibrated image can be printed and passed out to students as an introduction to measurement. The students would simply be given the sheets, told to work in small groups and allowed to have string and a millimeter ruler. Their goal is to determine the length of the cell. A discussion follows bearing on the accuracy of their results. A very recent plug-in from ReindeerGraphics makes it possible to directly calibrate the image you are working on. http: //reindeergraphics.com/ The same calibrated image can then be brought up under one of the following freeware programs; Scion Image (PC), http://www.scioncorp.com/ NIH Image Mac, or ImageJ. http://rsb.info.nih.gov/ij/

The application that is really coming into its own is ImageJ. This JAVA based applet can be used as a free-standing application or Incorporated into a WEB page. Students carrying out the above exercise in any of these applications can compare their results to what they received on the paper exercise. After calibrating their image, they can measure the area and perimeter of various structures and then compare these measurements to other ways of looking at the cheek cell like the SEM image shown in Figure 2[1]. A teacher using ImageJ can put class results on the internet and allow the students to interpret their results and write-up their conclusions
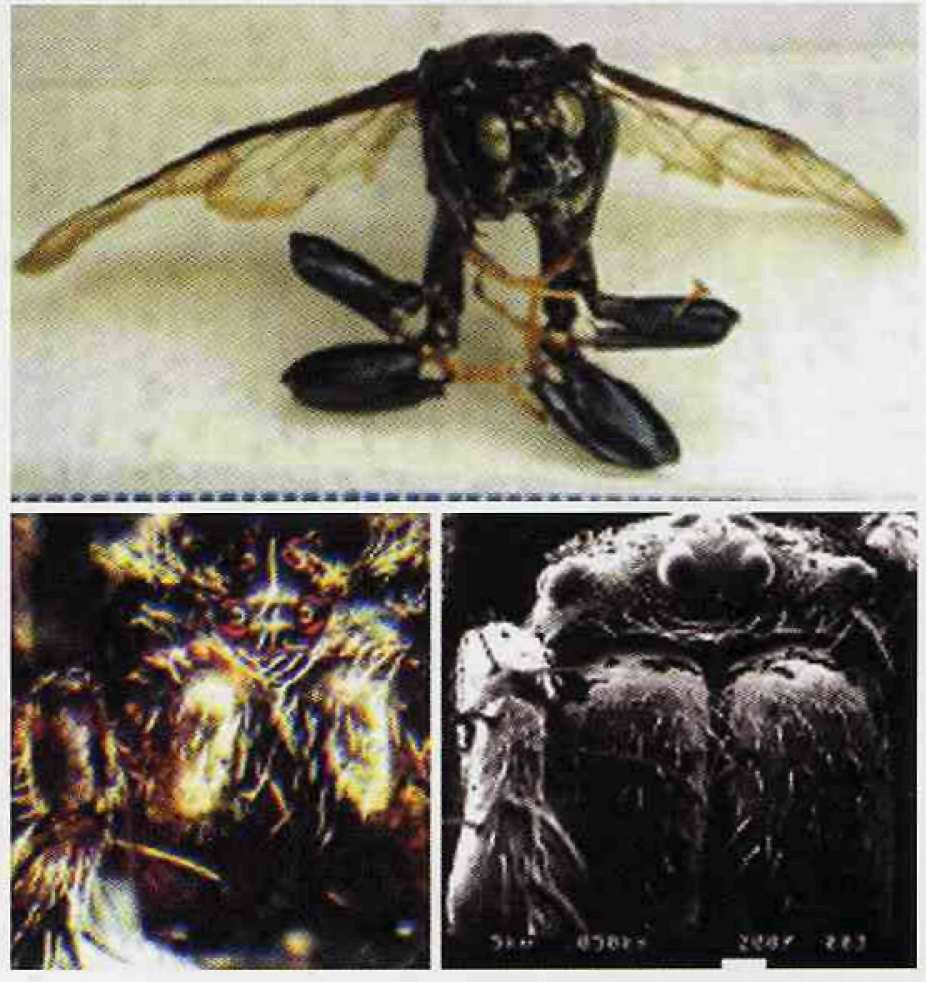

[Figure 4]. (Top) Elm Sawily

[Figure 5] (Bottom) LM and SEM view of Barn Spider. 


\section{Three Techniques, One PC, One System}

\section{Inca) - Fast, Integrated Microanalysis}

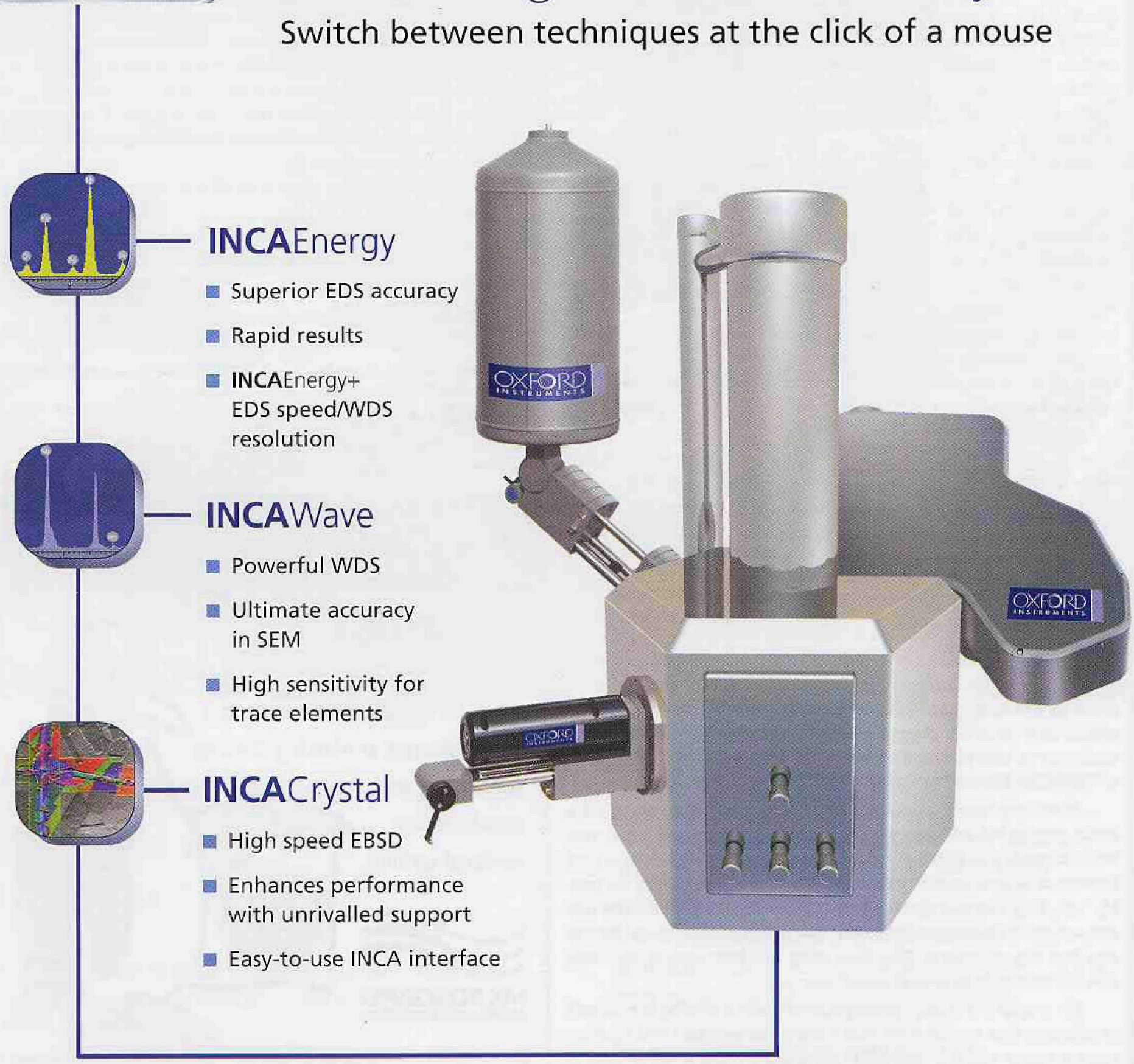

For your information pack on INCA, e-mail to:

info@ma.oxinst.com or contact us at the address below

Oxford Instruments Inc, 130A Baker Avenue Ext., Concord, MA 01742, USA.

Tel: +19783699933 Fax: +19783698287

www.oxford-instruments.com

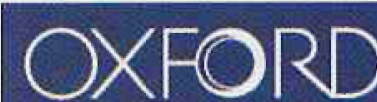




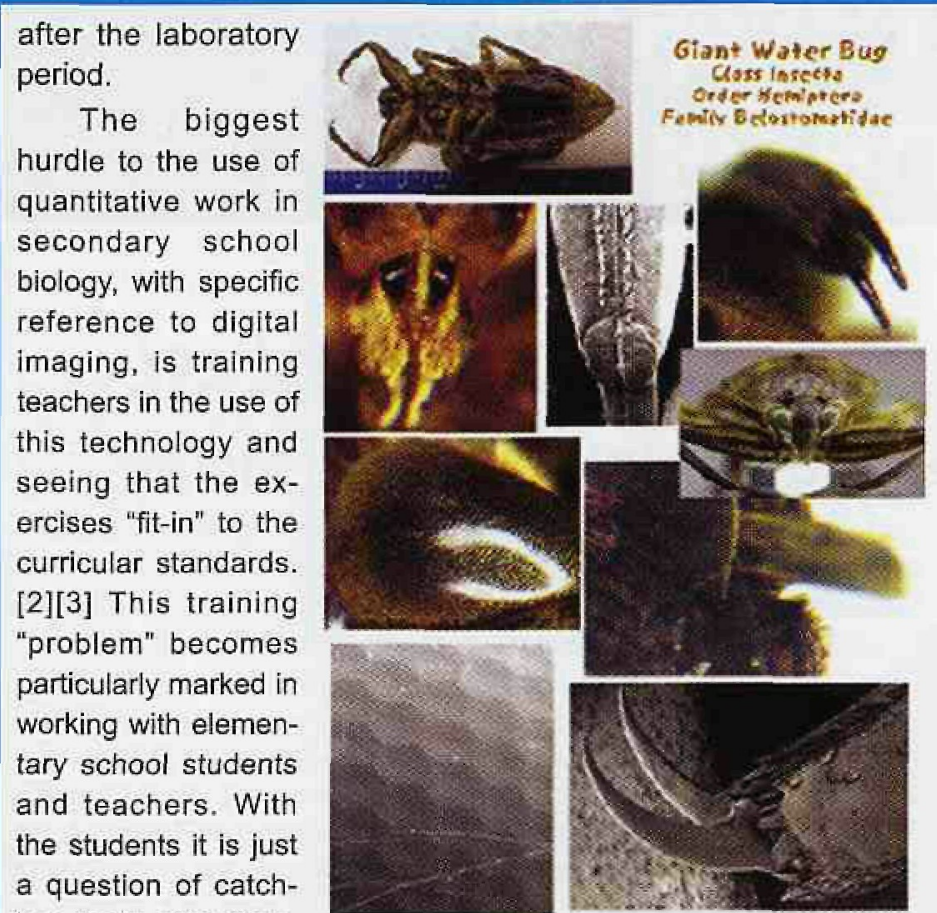

ing their attention.

However, with teachers it is trying to provide different levels of technology that they are are reasonably comfortable with. i.e. more paper and pencil exercises that lead to quantitative evaluations that can be made on the WEB.

The "Ugly Bug" contest that I ran this year was an attempt to introduce these same quantitative measuring skills into a K-8 science curriculum. Bugs are probably second only to dinosaurs in interest to most elementary students. In this contest I wanted to expand on some of the efforts that I had seen in a couple of state "Ugly Bug" contests [4][5] and include measuring exercises that the students could do with the bugs they collected. Subsequently they could compare their results with other school examples on the WEB.

The Educational Consultant for the State of New Hampshire agreed to mail out announcements of the contest to all elementary school principals. The announcement mailing included a description of contest prizes as well as a return postcard that a teacher in the school sent back announcing his or her classes' interest in participating. I e-mailed the teachers the rules and regulations for the contest and sent every class an "Ugly Bug Contest" poster to inspire them in their quest.

After members of a class collected several bugs they had to as a group choose the one they wanted to enter in the contest. Then they had to classify it as best they could. This invariably involved the school librarian or teacher assisting them in WEB searches to identify the bug. My "Ugly Bug" web site also furnished them with links to various groups and university databases. Some schools invited in parents and friends who had bug collections. The dead entry was then sent to me along with the classes best shot at classification.

On receipt of the bug I photographed it with a varying assortment of reflecting light microscopes plus a digital camera shot that incorporated a metric ruler for calibration purposes [Figure 4 (top)]. Typical digital photograph "Elm Sawfly" Then the mounted bug was dried, sputter coated and examined in the scanning electron microscope. I identified the submitted bugs and determined the winners. Emphasis was placed on the apparent effort that the students had made in classifying their entry.

At the end of the contest each participating school received an $11^{\prime \prime} \times 17^{\prime \prime}$ laminated poster of LM and SEM views of their bug [Figure
5]. They also received their "golden" bug back along with a couple of paper measuring exercises involving either a light microscope or SEM view of their entry. See a fine example of a student poster, left, from Stratham School, Stratham, $\mathrm{NH}$.

The class was directed to the Results WEB site[6] where they could see a LM view of their school's entry, plus all the other entries. On this same page was an ImageJ applet that came up and allowed students to view a variety of SEM bug images and answer the measurement questions.

The science supplier VWR had previous agreed to donate a good Stereo Microscope and two OK stereo microscopes as prizes. This took care of the first three prizes. All participating schools received laminated charts of various insects. The New Hampshire Science Teachers had a conference at Phillips Exeter at the end of March, 2002. I presented an imaging workshop at the conference and discussed the results of the contest with conference participants. On the basis of that feedback a decision was made to have an "Ugly Bug" contest next year. P.S. It was made clear at the end of the contest that there is no such thing as an ugly bug.

\section{References:}

[1] J. Ekstrom http://science.exeter.edu/jekstrom/WEB/CELLS/Epith/ Epith.html

[2] J.Ekstrom Cell Structure Study, The Science Teacher Vol 67, No. 7, October 2000 http://science.exeter.edu/jekstrom/nsta/elodea.html

[3] J. Ekstrom Slicing for Biology, The Science Teacher Vol 68, No. 2, Feb. 2001

[4] Marilee Sellers NAU "Ugly Bug" Contest http://www.nau.edu/\%7Eelectron/ bug/99/99.html

[5] Oklahoma Microscopy Society "Ugly Bug" contest http:// www.uglybug.org/

[6] New Hampshire "Ugly Bug" contest http://science.exeteredu/jekstrom/ BUGS/B.htm

\section{Psst. Buddy ... Need a Fluorescence Reference?}

\section{Fluor-Ref ${ }^{\text {Tm }}$}

Fluorescence Reference Slides

Great for Lamp centration, testing power settings, confirming staining consistency and standardizing confocal settings.

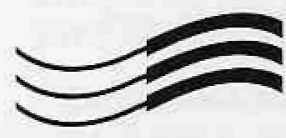
MICROSCOPY/ MICROSCOPY EDUCATION

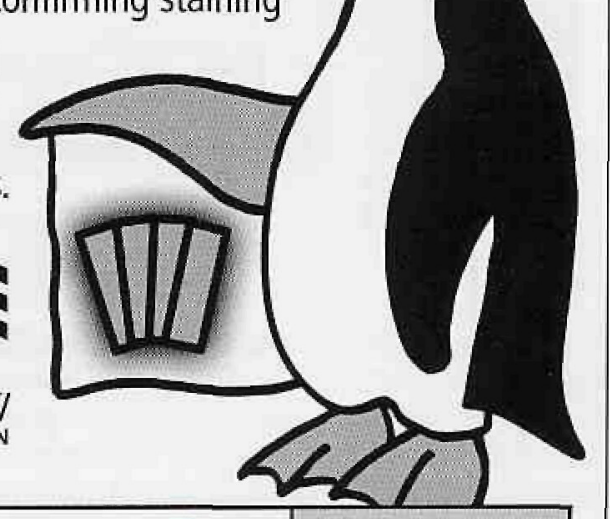

\begin{tabular}{|l|r|}
\hline Single slides & $\$ 18$ \\
\hline Set of $4(R, G, B / U V$, Orange $)$ & $\$ 55$ \\
\hline Classroom sets: 6 sets of 4 & $\$ 275$ \\
\hline
\end{tabular}

To Order: 413-746-6931 or www/MicroscopyEducation.com 


\section{for Specimen Preparation.}

lon Beam Sputter

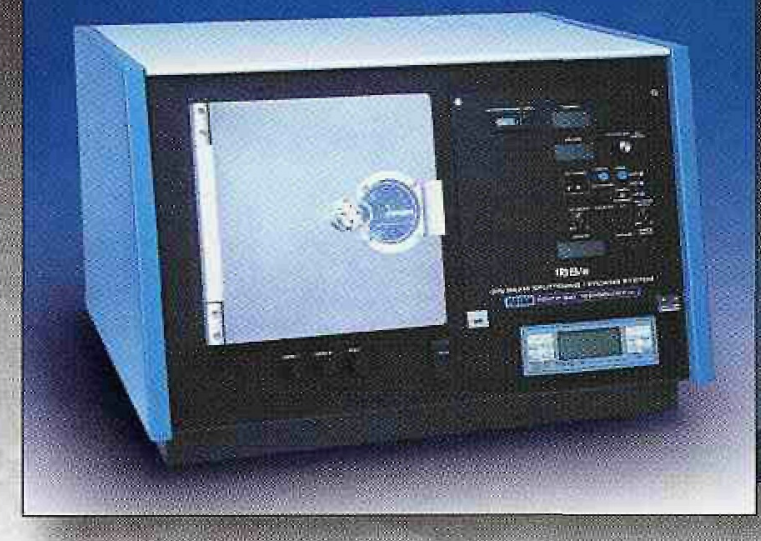
Deposition and Etching System

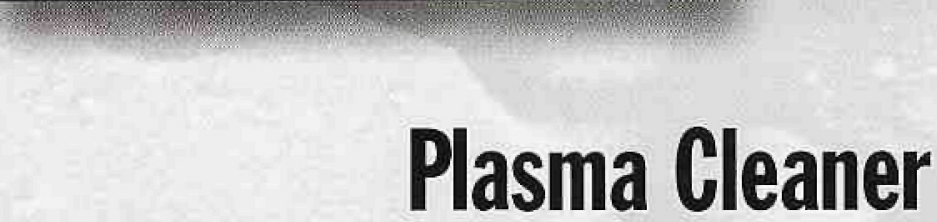

Removes hydrocarbons without damaging specimen 4 Intuitive front panel controls 4 Ideal for multi-user environments 4 Large chamber accommodates samples 4

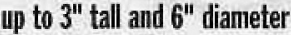

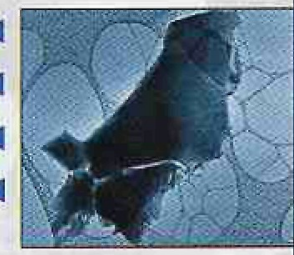

$>$ Uniformly thin, continuous, amorphous films

$>$ Multiple target materials without breaking vacum

I Improved imaging for highest magnification FESEM

$\checkmark$ Base pressure of $10^{-7}$ without $L H_{2}$ trapping

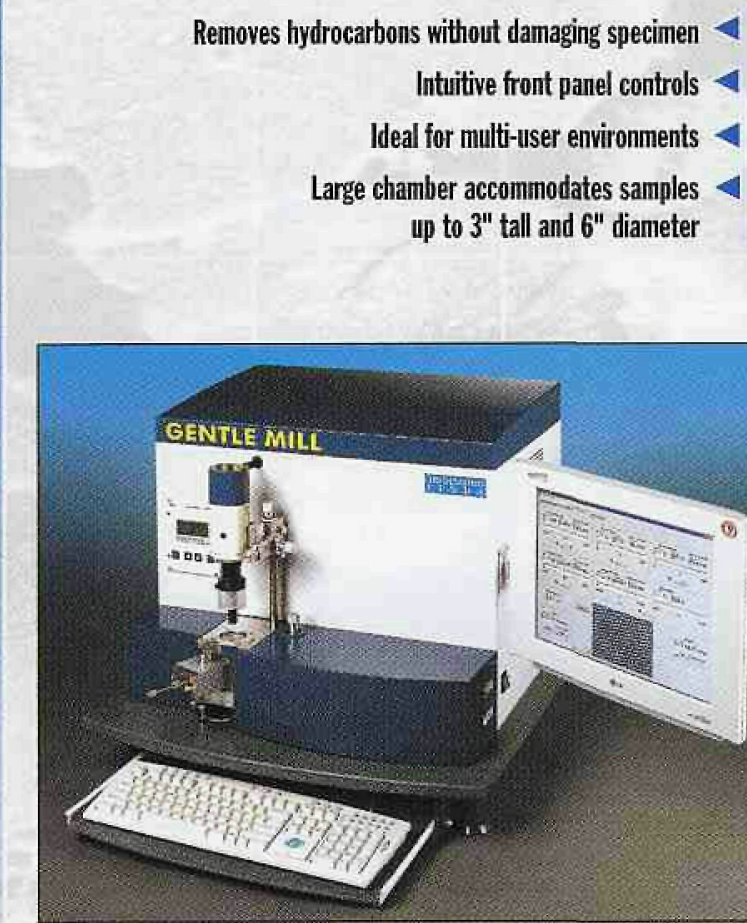

\section{Gentle Mill" Low Energy lon Polisher}

www.southbaytech.com

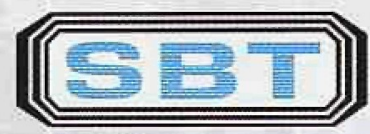

SOUTHEAY TECHWOLOEY INE

1120 Via Callejon | San Clemente, CA 92673 | U.S.A. | 949-492-2600 | Fax: 949-492-1499

Toll Free: 800-728-2233 | email: info@southbaytech.com | www.southbaytech.com 